anbiances Environnement sensible, architecture et espace urbain

\title{
Pascale Pichon et Fanny Herbert avec Alissone Perdrix (eds.). 2014. Atlas des espaces publics : Saint-Etienne, une ville laboratoire.
}

Publications de l'université de Saint-Etienne, Saint-Etienne, 2014, 296 pages, illustrations comprises.

Pascal Amphoux

\section{CpenEdition}

\section{Journals}

Édition électronique

URL : http://journals.openedition.org/ambiances/536

DOI : 10.4000/ambiances.536

ISSN : 2266-839X

Éditeur :

Direction Générale des Patrimoines - DAPA - MCC, UMR 1563 - Ambiances Architectures Urbanités (AAU)

\section{Référence électronique}

Pascal Amphoux, « Pascale Pichon et Fanny Herbert avec Alissone Perdrix (eds.). 2014. Atlas des espaces publics: Saint-Etienne, une ville laboratoire. », Ambiances [En ligne], Comptes-rendus, mis en ligne le 22 juin 2015, consulté le 22 septembre 2020. URL : http://journals.openedition.org/ ambiances/536 ; DOI : https://doi.org/10.4000/ambiances.536

Ce document a été généré automatiquement le 22 septembre 2020.

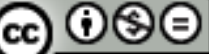

Ambiances is licensed under a Creative Commons Attribution-NonCommercial-NoDerivatives 4.0 International License. 


\section{Pascale Pichon et Fanny Herbert avec Alissone Perdrix (eds.). 2014. Atlas des espaces publics : Saint- Etienne, une ville laboratoire.}

Publications de l'université de Saint-Etienne, Saint-Etienne, 2014, 296 pages, illustrations comprises.

\section{Pascal Amphoux}

\section{RÉFÉRENCE}

Pascale Pichon et Fanny Herbert avec Alissone Perdrix (eds.). 2014. Atlas des espaces publics : Saint-Etienne, une ville laboratoire. Publications de l'université de SaintEtienne, Saint-Etienne, 2014, 296 pages, illustrations comprises.

1 Cet ouvrage collectif met en perspective huit années de travaux d'étudiants, menés principalement dans le cadre de la formation de master 2 «Espace public. Design, architecture, pratiques ", co-dirigé par des enseignants de l'Université Jean Monnet, de l'Ecole d'architecture (ENSASE) et de l'Ecole d'art et de design de Saint-Etienne (ESADSE). Principe premier? « Considérer Saint-Etienne comme un laboratoire en plein air » d'observation des mutations contemporaines de l'espace public et du paysage métropolitain. Second principe? Assembler les fragments d'évolution, d'observation ou de perception de l'espace public repérés pendant des années par des promotions d'étudiants successives, sous la forme d'un « atlas ", par définition inachevé, qui révèle, par fragments et sans prétention d'exhaustivité, «le visage contemporain et historiquement construit de la ville de Saint-Etienne, ville de migrations nées des ressources énergétiques du sol ». Troisième principe ? Une méthode reposant sur trois points fondamentaux: une approche sensible de l'espace public urbain croisant l'expérimentation in situ et l'enquête de terrain; l'extrapolation d'un répertoire de 
«qualités d'urbanité » propre à la ville de Saint-Etienne; et un dispositif de montage permettant de dire et d'imager ces qualités identitaires.

2 En résulte la structure en trois parties de l'ouvrage, intitulées métaphoriquement: " aborder », « cheminer », « se repérer ».

3 La première partie aborde donc le sujet, d'une part en faisant les présentations descriptives de l'ouvrage (textes de Pascale Pichon, Fanny Herbert et Alissone Perdrix qui précisent la suite des «contextes situés d'urbanité » qui ont été investigués, les méthodes proposées aux étudiants ainsi que les techniques de présentation et le mode d'emploi ou de lecture dudit atlas), d'autre part en réunissant des articles autonomes de plusieurs des enseignants ou personnalités invitées. Chacun de ces textes, savamment et richement référencié, a sa logique et sa qualité propre: y sont convoquées les notions d'«ambiance» (Jean-Paul Thibaud, sociologue), de " campement urbain " (Marie-Pierre Rolland, plasticienne) ou de " contemporanéité » (Christian Drevet, architecte) qui donnent en quelque sorte le paysage théorique dans lequel les auteurs s'inscrivent: une sorte de plaidoyer à la gloire des approches sensibles de l'espace public dont les tenants et aboutissants sont du coup bien cernés. Une mention particulière peut-être au texte de Yan Olivares qui introduit discrètement une notion de "dimension masquée» (et non "cachée») qui ne saurait être ni programmée, ni normalisée, ni strictement objectivée par une analyse scrupuleuse, mais qui demande justement à être démasquée dans et par le projet (l'auteur en fait même " la condition de la justesse de la conception en matière d'espace public »).

La deuxième partie est le corps cheminant de l'ouvrage (pp.56-266). Très richement illustrée, elle semble s'ordonner selon la suite des secteurs d'investigation proposés aux groupes d'étudiants aux différentes périodes et années d'enseignement. Elle se présente comme un corpus de soixante-treize planches, recomposées librement à partir de matériaux hétérogènes d'images et de textes qui mêlent systématiquement, selon des logiques de composition propres à chaque planche, une dizaine de types de signes ou d'informations : légendes précisant la méthode d'observation, titre de la planche, «matérialités » repérées, noms des lieux investigués, dates ou horaires d'observation, phrase off précisant la situation de l'usage observé, photographies plein cadre, un nuancier de couleurs remplaçant la numérotation des pages, un jeu de renvoi entre planches corrélées... De ce mélange résulte une lecture fouineuse, dans laquelle chacun pourra retrouver les bribes d'un savoir à reconstituer sur l'espace public stéphanois, savoir incomplet sans doute (c'est une revendication des auteurs), mais qui met le doigt sur une des dimensions recherchées : la dimension ordinaire de l'espace public - « rien d'exceptionnel, des fragments de vie quotidienne, des événements routiniers, des rencontres passagères ".

5 La troisième partie, intitulée se repérer, sous-entend que l'on se soit perdu en chemin dans le jeu des planches de la deuxième. Y sont en fait exposés et ordonnés : un répertoire des méthodes et outils offerts aux étudiants (une sorte de manuel définissant de manière synthétique et didactique des techniques aussi différentes que l'observation ethnographique, la marche urbaine, l'entretien, la recherche documentaire ou iconographique, le protocole expérimental, le scénario) ; un vocabulaire des «qualités d'urbanité de l'espace public » (six notions classiques y sont redéfinies, là aussi de manière cursive et claire: la mobilité, l'accessibilité, l'hospitalité, l'habitabilité, la visibilité, la citoyenneté); et quelques index des lieux, des «matérialités » ou des usages repérés, ainsi qu'une bibliographie. 
6 Le résultat est un grand livre volumineux dont il faudrait dire qu'il a les qualités de ses défauts - à moins que ce ne soit l'inverse. Pour la qualité donc, un témoignage, trop rare, d'une expérience d'enseignement qui s'échelonne et s'accumule sur plusieurs années. Nous sommes nombreux dans les écoles de grandes villes françaises à poursuivre des enseignements sur l'espace public pendant des années, dont nous ne prenons pas le temps de traiter le formidable corpus de connaissances ou de propositions que cela représente. Ce travail a ici été fait, avec courage et détermination. Et il faut y voir plusieurs avantages. A un premier niveau, cela constitue un hommage au travail collectif et une magnifique "parole rendue" à tous les participants qui ont donné la leur, sur plusieurs années à un titre ou à un autre, étudiant ou enseignant, autour de cette expérience d'enseignement. A un second niveau, il est évident que l'ouvrage constitue une bible de ressources et d'observations factuelles presqu'inépuisable pour tous les Stéphanois qui vivent ou travaillent sur l'espace public de la Ville (habitants ou concepteurs). A un troisième niveau, l'étudiant y trouvera des indications simples et didactiques sur les méthodes, les notions ou ce qu'il faudra désormais bientôt appeler les « idéologies » contemporaines des approches sensibles de l'espace public.

7 Mais ces qualités ont aussi leur revers. Pour celui qui n'a pas participé, le sentiment est plus mitigé. Il ne faut pas trop dire que le lecteur peut faire son propre cheminement dans l'ouvrage (après tout c'est un des droits fondamentaux de tout lecteur comme l'a montré Pennac en son temps), et la profusion des informations tous azimuts pourra au contraire donner un sentiment d'indigestion et rebuter par exemple le professionnel qui, même s'il s'intéresse au contenu, a besoin d'un peu d'assistance pour «faire un bout de chemin » justement et ne pas se réfugier dans les stéréotypes de la profession. De même, pour le concepteur ou le designer, l'explicitation laborieuse et complexe d'un montage somme toute assez simple de planches qui se révèlent finalement peu aimables, risque de le décourager... Et l'on peut tout de même reprocher à ce niveau un manque d'invention graphique ou d'ergonomie de lecture minimale : la rigidité de la forme et de la facture (dessins et écritures trop homogènes) n'ont pas la liberté d'un Latour, Hermant, Shannon (1998) dans un Paris ville invisible et ne laissent pas non plus, quoiqu'on en dise, le plaisir au lecteur de plonger " comme dans les cartes d'un atlas »: il ne suffit pas de dire que l'on pourra découvrir des terres inconnues ou des contrées lointaines (paroles habitantes ou parcours inédits), il faut encore trouver une forme de restitution graphique qui aide à produire cet effet, ce à quoi des étudiants en architecture peuvent être sensibilisés (l'effet ici est paradoxalement d'objectivation, qui refroidit en quelque sorte une matière pourtant riche). Quant au chercheur, il se pourrait bien qu'il reste sur sa faim - soit que, comme spécialiste de l'espace public, il n'en retrouve que les stéréotypes de représentation dans les théories ou pratiques contemporaines, soit que, comme militant des approches sensibles, il ne trouve matière à extraire de nouveaux principes méthodologiques, qu'il est pourtant en droit d'attendre de la liberté d'une expérimentation pédagogique.

8 Reste que ce sont là les revers d'un ouvrage qui, dans sa modestie louable, n'a peut-être d'autre ambition que d'avoir la valeur d'un témoignage heureux et d'un outil didactique - ce qu'il assume parfaitement et à de nombreux titres. 


\section{BIBLIOGRAPHIE}

Latour, Bruno ; Hermant, Emilie \& Shannon, Susanna. 1998. Paris ville invisible. Paris : Les empêcheurs de penser en rond / La découverte.

\section{AUTEURS}

\section{PASCAL AMPHOUX}

Pascal AMPHOUX. Architecte et géographe. Professeur à l'Ecole Nationale Supérieure d'Architecture de Nantes. Activité indépendante de praticien et de consultant sur de nombreux projets architecturaux, urbains et/ou environnementaux (Bureau CONTREPOINT, Projets urbains, Lausanne). Chercheur au Centre de Recherches sur l'Espace Sonore et l'Environnement Urbain (CRESSON, Ecole Nationale Supérieure d'Architecture de Grenoble, UMR 1563 AAU). Membre du conseil scientifique Europan Europe, expert auprès de diverses institutions suisses, françaises ou européennes. Auteur de nombreux ouvrages et publications scientifiques portant sur l'environnement sonore, et de façon plus spécifique, sur les rapports entre la pratique du projet, l'esthétique paysagère et les méthodes des sciences sociales.

Mail : pascal.amphoux@sunrise.ch 